\title{
Prevalência de insuficiência venosa superficial dos membros inferiores em pacientes obesos e não obesos
}

\author{
Prevalence of lower limbs superficial venous insufficiency in obese and non-obese patients \\ Amélia Cristina Seidel', Amanda Sampaio Mangolim², Leandro Pablos Rossetti², Juliana Regina Gomes², Fausto Miranda Jr
}

\begin{abstract}
Resumo
Contexto: A insuficiência venosa crônica dos membros inferiores é a mais prevalente das doenças venosas. Muito se discute sobre sua etiologia e fisiopatologia. Vários fatores de risco têm sido associados ao seu desenvolvimento, como idade, sexo, dieta, entre outros. A obesidade é um problema de saúde pública e sua incidência tem aumentado. O ecocolor Doppler é um método útil para avaliar a presença de refluxo e/ou obstrução no sistema venoso.

Objetivo: Comparar a prevalência de insuficiência venosa superficial e sintomas associados em pacientes obesos e não obesos.

Métodos: Após pesagem, medição da estatura e exame físico, os pacientes com índice de massa corpórea (IMC) $<30 \mathrm{~kg} / \mathrm{m}^{2}$ e $>35 \mathrm{~kg} / \mathrm{m}^{2}$ e queixas compatíveis com insuficiência venosa foram distribuídos nos grupos I e II, respectivamente. Foram submetidos à realização do ecocolor Doppler dos membros inferiores para avaliação da presença ou não de refluxo.

Resultados: Foram examinados 311 membros de 168 pacientes com 25-72 anos. Para análise estatística, foram consideradas queixas de varizes, dor, edema, dermatite, eczema e úlcera, associados ou não. Foi obtido um total de 109 e 104 membros com varizes nos grupos I e II, respectivamente. Queixas de varizes visíveis $(p<0,001)$ e varizes visíveis com dor $(p=0,0118)$ foram mais prevalentes no grupo I. Queixas de varizes com edema $(p<0,001)$, somente edema $(p<0,001)$ e edema associado a dor $(p<0,001)$ foram mais prevalentes no grupo II. Os dados não mostraram diferença estatisticamente significante na prevalência de varizes entre os grupos I e ll.

Conclusão: A prevalência de varizes é semelhante entre os obesos e não obesos; as queixas clínicas diferem entre os grupos e são compativeis e dependentes da presença de insuficiência venosa.
\end{abstract}

Palavras-chave: Insuficiência venosa; veia safena; obesidade; extremidade inferior.

\begin{abstract}
Background: Chronic venous insufficiency of the lower limbs is the most prevalent venous disease. There is an ongoing debate about its etiology and pathophysiology. Several risk factors have been associated with its development, such as age, sex and diet. Obesity is a public health problem and its prevalence has been increasing. Color Doppler ultrasonography is a useful method to evaluate the presence of reflux and/or obstruction of the venous system.

Objective: To compare the prevalence of superficial venous insufficiency and associated symptoms in obese and non-obese patients.

Methods: After weighing, height measurement and physical examination, patients with body mass index (BMI) $<30 \mathrm{~kg} / \mathrm{m}^{2}$ and $>35 \mathrm{~kg} / \mathrm{m}^{2}$ and complaints compatible with venous insufficiency were divided into groups I and II, respectively. They underwent color Doppler ultrasonography of the lower limbs, in order to assess the presence or absence of reflux.

Results: A total of 311 limbs of 168 patients 25 to 72 years old were examined. For statistical analysis, complaints of varicose veins, pain, swelling, dermatitis, eczema and ulcers, associated or not, were considered. A total of 109 and 104 limbs with varicose veins were obtained in groups I and II, respectively. The prevalence of visible varicose veins $(p<0.001)$ and visible varicose veins with pain $(p=0.0118)$ was higher in group I. Complaints of varicose veins with edema $(p<0.001)$, only edema $(p<0.001)$ and edema with pain $(p<0.001)$ were more prevalent in group II. Data did not present significant statistical difference in the prevalence of varicose veins between groups I and II.

Conclusion: The prevalence of varicose veins in obese and non-obese individuals is similar; clinical complaints differ between groups and are consistent and dependent on the presence of venous insufficiency.
\end{abstract}

Keywords: Venous insufficiency; saphenous vein; obesity; lower extremity.

Trabalho realizado na Disciplina de Angiologia e Cirurgia Vascular dos Cursos de Medicina da Universidade Estadual de Maringá (UEM) e da Universidade Federal de São Paulo (UNIFESP). 'Professora Associada da Disciplina de Angiologia e Cirurgia Vascular do Curso de Medicina da Universidade Estadual de Maringá (UEM), Maringá (PR), Brasil; Ultrassonografista Vascular pela Sociedade Brasileira de Angiologia e de Cirurgia Vascular (SBACV) e pelo Colégio Brasileiro de Radiologia (CBR).

${ }^{2}$ Acadêmico do $4^{\circ}$ ano de Medicina da UEM, Maringá (PR), Brasil.

${ }^{3}$ Professor Titular da Disciplina de Cirurgia Vascular do Departamento de Cirurgia da Universidade Federal de São Paulo (UNIFESP); Ultrassonografista Vascular pela SBACV e CBR.

Não foram declarados conflitos de interesse associados à publicação deste artigo.

Submetido em: 04.01.11. Aceito em: 12.04.11.

J Vasc Bras. 2011;10(2):124-130 


\section{Introdução}

A insuficiência venosa crônica (IVC) dos membros inferiores (MMII) é a mais prevalente das doenças venosas $^{1}$. No século 20 , numerosas teorias foram propostas em relação à etiologia e fisiopatologia da IVC, sendo mais discutida aquela que se refere à hipertensão secundária ao refluxo e/ou à obstrução no sistema venoso ${ }^{2}$. Essas anormalidades causam dor e desconforto no membro afetado e podem eventualmente evoluir para formação de úlceras de estase venosa.

Vários fatores de risco têm sido associados ao desenvolvimento da insuficiência venosa, como obesidade, idade, sexo, estilo de vida, trabalho, dieta, uso de hormônios, gravidez, história familiar e muitos outros ${ }^{3-5}$.

A obesidade é o maior problema de saúde pública em todos os países industrializados e desenvolvidos. Nesses países, estima-se que $1 / 3$ da população tem obesidade grave que requer tratamento para evitar o desenvolvimento de doenças e complicações graves como hipertensão arterial (HA), diabetes mellitus (DM), stress respiratório, doenças venosas com úlceras de MMII entre outras ${ }^{6}$. É importante salientar que, além dos países industrializados e desenvolvidos, a obesidade afetando adultos e crianças também está presente em nações em desenvolvimento ${ }^{7}$.

A história clínica e um meticuloso exame físico fornecem o diagnóstico de IVC grave devido à presença de suas características como hiperpigmentação, edema, úlceras ativas ou cicatrizadas, mas na ausência de alterações de pele haverá necessidade de exames complementares para o diagnóstico preciso ${ }^{8}$.

Recentes avanços no laboratório vascular permitem acesso à incompetência dos sistemas venosos superficial $\mathrm{e}$ profundo, tão bem quanto a avaliação da função venosa da perna, não somente de pacientes com doença venosa, mas também em pessoas que são assintomáticas ou não têm sinais visíveis da doença venosa ${ }^{9}$.

O ecocolor Doppler é, atualmente, o método mais útil para avaliação da incompetência das válvulas venosas ou obstrução crônica ${ }^{3,8}$. É um método rápido, não invasivo, de custo razoável $^{3}$ e apresenta $92 \%$ de sensibilidade e $73 \%$ de especificidade na detecção do refluxo quando comparado a flebografia descendente ${ }^{8}$.

Devido ao aumento da incidência da obesidade na população e sabendo-se que esta frequentemente apresenta queixas compatíveis com insuficiência venosa de MMII, este trabalho teve como objetivo comparar a prevalência de insuficiência venosa superficial e sintomas associados em pacientes obesos e não obesos.

\section{Métodos}

Em um período de 5 anos, foram avaliados 168 pacientes encaminhados ao Laboratório de Ultrassonografia Med Imagem, Maringá (PR), com queixas compatíveis com insuficiência venosa. O protocolo do estudo foi aprovado pelo comitê de ética da Universidade Estadual de Maringá (UEM) e, após assinarem o termo de consentimento informado, todos os pacientes foram pesados $(\mathrm{kg})$ e medidos $(\mathrm{m})$ e esses valores foram usados para cálculo do índice de massa corpórea (IMC) de cada um, de acordo com a fórmula: IMC $=\left[\right.$ peso $/$ altura $\left.^{2}\right]$. É um estudo prospectivo de corte transversal de prevalência. A formação da amostra foi feita de forma aleatória, com pareamento dos pacientes por sexo e faixa etária, e distribuída em dois grupos, sendo que cada um ficou com o mesmo número de pacientes dos dois sexos em cada faixa etária. O grupo I, com 84 pacientes que tinham IMC $<30 \mathrm{~kg} / \mathrm{m}^{2}$, e o grupo II, com outros 84 pacientes, com IMC $>5 \mathrm{~kg} / \mathrm{m}^{2}$.

Após anamnese e exame físico, os pacientes foram submetidos ao exame de ecocolor Doppler, cujos achados foram anotados em uma folha estruturada (protocolo) para posterior análise.

O exame foi realizado com um aparelho de ultrassom (Philips EnVisor, Philips Medical System, EUA) utilizandose transdutores para imagem de 7,5 e $10 \mathrm{MHz}$, permitindo a visibilização dos sistemas venosos profundo (veias femoral, poplítea, tibiais posteriores e fibulares) e superficial (veia safena magna (VSM), veia safena parva (VSP) e tributárias) e das veias perfurantes, conforme a técnica descrita na literatura Foi considerado segmento com refluxo quando havia fluxo reverso com tempo maior que 0,5 segundos e velocidade maior que $30 \mathrm{~cm} / \mathrm{segundos}$.

Os critérios de exclusão para este estudo foi a presença de insuficiência venosa profunda, insuficiência arterial, diabetes mellitus, hipertensão arterial e história de operação venosa prévia.

Análise estatística dos resultados foi realizada com nível de confiança de $95 \%$ utilizando-se o teste do qui-quadrado, mas quando as frequências esperadas foram baixas (menores que 5), não sendo esse teste apropriado, optou-se pela utilização do teste Exato de Fisher. O software utilizado foi o R2.12.2.

\section{Resultados}

Trezentos e onze membros de 168 pacientes foram examinados com clínica de insuficiência venosa de MMII, 
sendo 126 (75\%) pacientes do sexo feminino e 42 (25\%) do sexo masculino, com idade entre 25 e 72 anos (Tabela 1).

Para análise estatística entre os grupos, foram consideradas as seguintes queixas dos pacientes: presença de varizes, dor, edema, dermatite ocre (consideradas manchas castanhas na pele), lipodermatoesclerose (endurecimento com retração fibrótica), eczema e úlcera, associados ou não (Tabela 2).

A Tabela 3 e as Figuras 1 e 2 mostram o resultado da avaliação das veias superficiais quanto à presença ou não de refluxo, considerando-se as veias tronculares (VSM e VSP), suas tributárias, as veias perfurantes e suas associações.

Totalizando os membros com refluxo superficial, foi observado que no grupo I havia 109 membros com varizes e 52 sem varizes; e no grupo II, 104 membros com varizes

Tabela 1. Distribuição dos 168 pacientes com clínica de insuficiência venosa de membros inferiores conforme sexo e faixa etária

\begin{tabular}{lcc}
\hline Idade & Feminino & Masculino \\
\hline $20-29$ anos & 4 & 3 \\
$30-39$ anos & 6 & 4 \\
$40-49$ anos & 16 & 5 \\
$50-59$ anos & 27 & 2 \\
$60-69$ anos & 11 & 2 \\
$70-79$ anos & 3 & 1 \\
Total & 67 & 17 \\
\hline
\end{tabular}

Tabela 2. Manifestações clínicas relacionadas aos membros dos pacientes distribuídos nos grupos de acordo com o índice de massa corpórea (IMC)

\begin{tabular}{lccc}
\hline \multirow{2}{*}{ Manifestações clínicas } & Grupo I & Grupo II & \multirow{2}{*}{ Valor de p } \\
\cline { 2 - 3 } & IMC $<30$ & IMC $>35$ & \\
\hline Varizes & 112 & 20 & $<0,001^{*}$ \\
Varizes + dor & 15 & 3 & $0,0118^{*}$ \\
Varizes + edema & 0 & 22 & $<0,001^{*}$ \\
Varizes + dermatite & 0 & 3 & 0,1110 \\
Varizes + eczema & 0 & 1 & 0,4823 \\
Varizes + edema + eczema + dermatite & 0 & 1 & 0,4823 \\
Varizes + edema + dor & 2 & 7 & 0,1438 \\
Edema & 0 & 36 & $<0,001^{*}$ \\
Edema + dermatite & 0 & 1 & 0,4823 \\
Edema + dermatite + eczema & 0 & 1 & 0,4823 \\
Edema + dor & 4 & 32 & $<0,001^{*}$ \\
Edema + dor + dermatite & 0 & 2 & 0,2318 \\
Edema + dermatite + eczema + úlcera & 0 & 1 & 0,4823 \\
Edema + úlcera & 0 & 1 & 0,4823 \\
Dor & 28 & 18 & 0,1808 \\
Úlcera & 0 & 1 & 0,4823 \\
Total do número de membros & 161 & 150 & \\
*p<0,05. & & & \\
\hline
\end{tabular}

${ }^{*} p<0,05$. enquanto que 46 membros não tinham varizes, apesar da clínica compatível (Figura 3).

Analisando estatisticamente esses resultados, verificou-se que as queixas de presença de varizes visíveis $(\mathrm{p}<0,001)$ e varizes visíveis associadas a dor $(\mathrm{p}=0,0118)$ foram mais prevalentes no grupo I. As queixas de varizes somadas a edema $(p<0,001)$, somente edema $(p<0,001)$ e edema associado a dor ( $\mathrm{p}<0,001)$ foram mais prevalentes no grupo II. Os dados não mostraram diferença estatisticamente significante na prevalência de varizes entre o grupo I e II ( $p=0,757)$, apesar de haver diferença entre as veias acometidas (Tabela 3 ).

Diante desses dados, verifica-se que as queixas dos pacientes eram compatíveis e dependentes da presença de insuficiência venosa, apesar de a clínica manifestada ser diferente entre os dois grupos.

Tabela 3. Veias do sistema venoso superficial com refluxo em relação ao número de membros dos pacientes distribuídos de acordo com o índice de massa corpórea (IMC)

\begin{tabular}{|c|c|c|c|}
\hline \multirow{2}{*}{ Veia } & Grupo I & Grupo II & \multirow{2}{*}{ Valor $\mathrm{p}$} \\
\hline & $\mathrm{IMC}<30$ & $I M C>35$ & \\
\hline VSM & 13 & 10 & 0,6354 \\
\hline VSM + perfurante & 7 & 24 & $0,0006^{*}$ \\
\hline VSM + tributária VSP & 3 & 5 & 0,4889 \\
\hline VSM + perfurante + tributária VSP & 3 & 5 & 0,4889 \\
\hline VSP & 2 & 1 & 1 \\
\hline VSP + perfurante & 2 & 2 & 1 \\
\hline VSP + tributária VSM & 1 & 0 & 1 \\
\hline VSP + perfurante + tributária VSM & 1 & 1 & 1 \\
\hline$V S M+V S P$ & 2 & 0 & 0,4990 \\
\hline Perfurante & 8 & 15 & 0,0502 \\
\hline $\begin{array}{l}\text { Perfurante + tributária VSM + } \\
\text { tributária VSP }\end{array}$ & 3 & 3 & 1 \\
\hline Tributária VSM & 25 & 16 & 0,2025 \\
\hline Tributária VSP & 11 & 9 & 0,7650 \\
\hline VSM + VSP + perfurante & 2 & 2 & 1 \\
\hline Perfurante + tributária VSM & 7 & 5 & 0,6425 \\
\hline Perfurante + tributária VSP & 5 & 2 & 0,4499 \\
\hline Tributária VSM + tributária VSP & 14 & 54 & $<0,001^{*}$ \\
\hline Sem varizes & 52 & 46 & 0,7570 \\
\hline Total do número de membros & 161 & 150 & \\
\hline
\end{tabular}

VSM: veia safena magna; VSP: veia safena parva; ${ }^{*} \mathrm{p}<0,05$ 


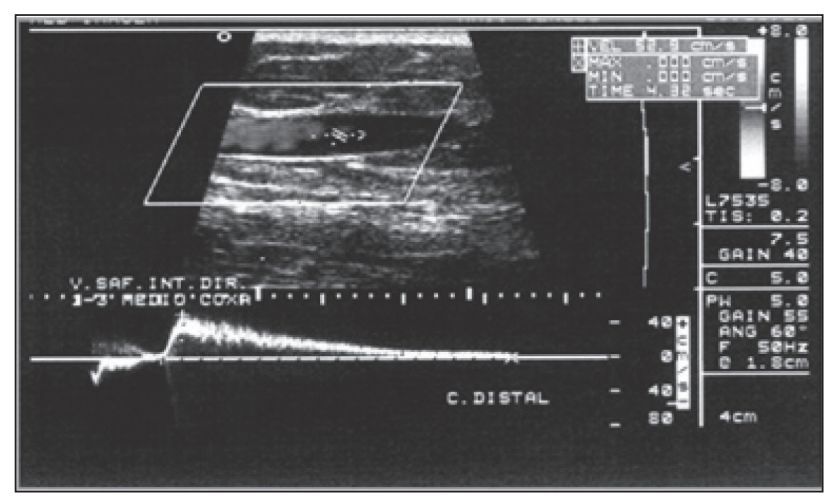

Figura 1. Ecocolor Doppler mostrando sistema venoso com refluxo.

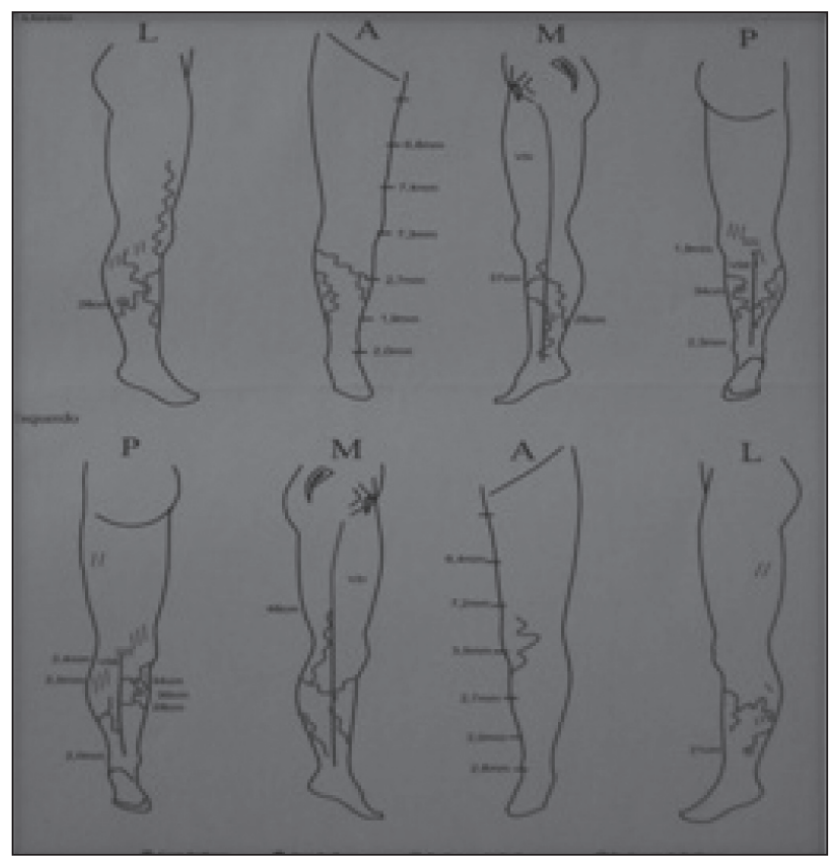

Figura 2. Desenho após mapeamento das veias com refluxo.

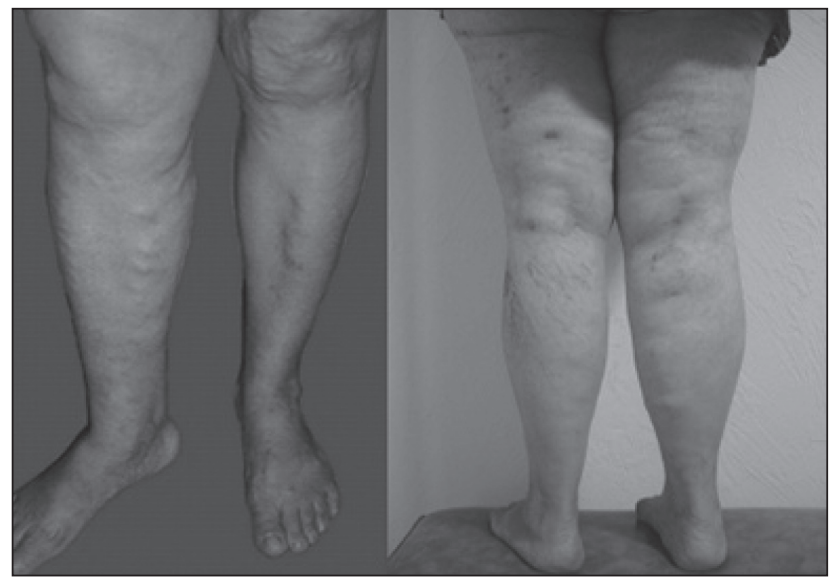

Figura 3. Fotografia de membros de pacientes dos grupos I e II.

\section{Discussão}

$\mathrm{Na}$ amostra estudada, o grupo II foi formado somente com pacientes com IMC $>35 \mathrm{~kg} / \mathrm{m}^{2}$, apesar de já serem considerados como obesos aqueles que apresentam um valor do IMC $>30 \mathrm{~kg} / \mathrm{m}^{2}$, de acordo com a descrição citada no artigo de Ageno et al. ${ }^{8}$ e também pela definição da Organização Mundial da Saúde (OMS), citada no artigo de Jawien?.

Canadian guidelines ${ }^{10}$ distribuem os obesos em três classes, sendo: classe I formada por indivíduos com IMC entre 30 e $34,9 \mathrm{~kg} / \mathrm{m}^{2}$; classe II, IMC entre 35 e $39,9 \mathrm{~kg} /$ $\mathrm{m}^{2}$; classe III, IMC $>40 \mathrm{Kg} / \mathrm{m}^{2}$. Dessa maneira, neste estudo foram considerados os obesos das classes II e III.

O exame clínico dos pacientes foi realizado com os mesmos em pé para inspeção e análise de veias tronculares e tributárias, como sugerido por Iannuzzi et al. ${ }^{11}$.

As varizes são consideradas uma parte do espectro da IVC de acordo com os critérios de Basle citados por Beebe-Dimmer et al. ${ }^{12} \mathrm{e}$, neste trabalho, foram consideradas somente as varizes tronculares, definidas como troncos tortuosos e dilatados da VSM ou VSP e seus ramos de primeira e segunda ordem associada ou não à presença de veias perfurantes.

Há importantes variações na interpretação dos resultados dos estudos epidemiológicos e dificuldades na comparação deles por três fatores: porque são usadas muitas definições de veias varicosas, pela presença de técnicas de medidas diferentes entre os estudos e devido aos grupos ocupacionais terem sido mais bem estudados do que a população geral ${ }^{9}$.

Recentemente, clínicos e pesquisadores têm creditado o uso do ecocolor Doppler para detectar incompetência valvular associada com $\mathrm{IVC}^{13-16}$, sendo esse exame útil para pacientes obesos com sintomas de IVC, mas sem sinais clínicos claros de varicosidades ${ }^{17}$. Neste trabalho, o exame de ecocolor Doppler foi realizado conforme a técnica citada na literatura ${ }^{18}$.

Há alguns trabalhos que se referem à prevalência de varizes relacionando-a com certos fatores de risco, sendo a obesidade o objetivo nesta amostra.

Na prática clínica, pacientes para tratamento de veias varicosas geralmente são jovens, mulheres, têm história de flebite e frequentemente têm história familiar de veias varicosas.

Semelhantemente aos trabalhos revisados ${ }^{9,11,12,19,20}$, foi encontrado um predomínio do sexo feminino (75\%), havendo, porém, diferença em relação à idade, com maior predomínio de pacientes entre 50 e 59 anos (34,5\%). 
Estudando a epidemiologia de veias varicosas e IVC em relação aos fatores de risco, uma revisão da literatura (1942-2004) citou, além de outros, a obesidade como um desses fatores associados ao desenvolvimento de veias varicosas, apesar de também ressaltarem a dificuldade da comparação dos resultados devido às diferentes definições utilizadas na classificação dessas veias ${ }^{12}$.

Sugerman et al. ${ }^{21}$ e Padberg et al..$^{22}$ também afirmaram que a obesidade grave está associada com riscos de estase venosa em MMII, ulceração pré-tibial, celulite e edema bronze e, segundo Porter e Moneta ${ }^{23}$, apesar da associação comum com insuficiência venosa, a obesidade ainda não foi incorporada no sistema de classificação CEAP.

Nos obesos, o aumento da pressão intra-abdominal tem sido relacionado como fator causal na IVC, pois leva a um maior refluxo, aumento do diâmetro da veia e da pressão venosa ${ }^{24}$ e também diminui a vis a front, que é um dos fatores centrípetos mais importantes na fisiologia do retorno venoso. Além disso, a falta de atividade física nessas pessoas por diminuição da atuação da bomba muscular da panturrilha poderia em parte ser responsável pela falta de esvaziamento venoso da perna ${ }^{25,26}$.

De acordo com Sugerman et al., fica uma dúvida: a insuficiência venosa grave tem etiologia diferente na obesidade mórbida? É venosa? Em seu estudo, encontraram pacientes com obesidade classe III com sintomas graves nos membros, típicos de IVC, mas aproximadamente $2 / 3$ dos membros não tinham evidência anatômica de doença venosa; assim, indagaram se a associação do aumento dos sintomas nos membros com a obesidade sugeria que esta contribui para a morbidade ${ }^{21}$.

Estudos como os de Framingham ${ }^{27}$, Edinburg ${ }^{28}$ e muitos outros ${ }^{11,29-33}$ sugeriram uma significante associação entre veias varicosas tronculares, peso e IMC somente na mulher, não havendo indicação consistente de que essa relação seja verdadeira para o homem ${ }^{34,35}$.

A opinião permanece dividida se a obesidade por si é um fator de risco. Parece que não há associação entre obesidade e veias varicosas no homem e que a evidência para tal associação na mulher é confusa, estando presente em suas formas mais graves. Parece também pouco provável que a obesidade seja um fator de risco em um sexo e não no outro; talvez a obesidade possa agir como promotor de veias varicosas mais do que como um fator de risco primário verdadeiro $^{7}$, ou seja, ela agravaria um quadro pré-existente ${ }^{36,37}$.

Em um trabalho no qual os sintomas mais graves de IVC nos MMII aumentaram em gravidade (CEAP e VCSS) com a magnitude da obesidade (IMC), aproximadamente 1/3 dos membros estudados não tinha evidência anatômica de doença venosa, e nenhuma relação com achados do ultrassom foi observada. Os dados sugeriram que a classe III da obesidade mórbida seria considerada o maior fator contribuinte para a gravidade dos sintomas, sendo a origem venosa ou não ${ }^{22,24}$.

Uma das explicações alternativas para essa discrepância é a hipótese de que a obesidade maciça provavelmente produziria uma relativa obstrução ao retorno venoso dos MMII, semelhantemente ao efeito da ascite, ainda que o efeito combinado do aumento da demanda cardíaca possa ser adicionado à diminuição do retorno venoso, produzindo disfunção arteriovenosa central. Outra causa provável seria a obstrução linfática, na qual episódios repetidos de celulite poderiam levar a cicatrização dos vasos linfáticos. Talvez seja um efeito combinado de hipertensão linfovenosa ${ }^{22}$.

Mulheres com IMC $>30 \mathrm{~kg} / \mathrm{m}^{2}$ após menopausa apresentaram uma maior prevalência de veias varicosas, e a possível explicação deste achado é que a obesidade poderia dificultar o fluxo de sangue normal trocado entre veias superficiais e profundas do MI devido ao aumento da adiposidade e tecido fibroso circundando as veias, o que causaria um distúrbio na circulação venosa cutânea e dano às veias de drenagem, provocando estase e, subsequentemente, o aparecimento de veias varicosas ${ }^{38}$.

Outros fatores, tal como a falta de atividade física nas pessoas obesas, poderiam em parte ser responsáveis pela falta de esvaziamento venoso da perna, e a terapia de compressão para diminuir a hipertensão venosa é certamente mais difícil para aplicar efetivamente em pernas grandes e poderia contribuir para alta incidência de doença mais grave nos pacientes obesos ${ }^{25,39}$. Por outro lado, é possível que pacientes com IVC e veias varicosas sejam menos ativos fisicamente devido a sua condição e, por isso, mais predispostos a tornarem-se obesos ${ }^{12}$.

As queixas de 168 pacientes (311 membros) eram compatíveis com IVC, isto é, referiam principalmente presença de varizes (112 membros no grupo I e 30 membros no grupo II) e edema (36 membros no grupo II), associados ou não a outros sinais ou sintomas; destes, 98 membros $(31,5 \%)$ não tinham refluxo superficial, sendo 52 membros do grupo I e 46 do grupo II.

Após análise dos resultados, foi constatado que a VSM estava comprometida em 26 e 44 membros nos grupos I e II, respectivamente; a VSP estava comprometida em 6 e 4 membros nos grupos I e II, respectivamente; e VSM mais VSP estavam comprometidas em 2 membros no grupo I, estando ou não associadas a outras fontes de refluxo. Considerando-se ainda outras veias superficiais com refluxo, foi obtido um total de 213 membros $(68,5 \%)$ com varizes. 
Esta análise é limitada ao grupo de pacientes sintomáticos previamente examinados por especialistas e encaminhados para o estudo ultrassonográfico venoso. Não é aplicável a uma população selecionada somente pelo IMC, o que, possivelmente, demandaria outro estudo.

Foi possível concluir que a prevalência de varizes é semelhante entre obesos e não obesos; já as queixas clínicas diferem entre os grupos e são compatíveis e dependentes da presença de insuficiência venosa.

\section{Referências}

1. Abu-Own A, Scurr JH, Coleridge Smith PD. Saphenous vein reflux without incompetence at the saphenousfemoral junction. $\mathrm{Br}$ J Surg. 1994;81:1452-4.

2. Pappas PJ, Lal BK, Cerveira J), Padberg FT Jr, Duran WN. Causes of severe chronic venous insufficiency. Semin Vasc Surg. 2005;18:30-5.

3. Labropoulos N, Leon LR Jr. Duplex evaluation of venous insufficiency. Semin Vasc Surg. 2005;18:5-9.

4. Melissas J, Christodoulakis M, Spyridakis M, et al. Disorders associated with clinically severe obesity: significant improvement after surgical weight reduction. South Med J. 1998;91:1143-8.

5. Buchwald $H$. Overview of bariatric surgery. J Am Coll Surg. 2002;194:367-75.

6. Bergan JJ, Pascarella L. Severe Chronic venous insufficiency: primary treatment with sclerofoam. Semin Vasc Surg. 2005;18:49-56.

7. Callam MJ. Epidemiology of varicose veins. Br J Surg. 1994;81:16773.

8. Ageno W, Piantanida E, Dentali F, et al. Body mass index is associated with the development of the post-thrombotic syndrome. Thromb Haemost. 2003;89:305-9.

9. Jawien $\mathrm{A}$. The influence of environmental factors in chronic venous insufficiency. Angiology. 2003;54:S19-31.

10. Douketis JD, Paradis G, Keller H, Martineau C. Canadian guidelines for body weight classification in adults: application in clinical practice to screen for overweight and obesity and to assess disease risk. CMAJ. 2005; 172:995-8.

11. lannuzzi A, Panico S, Ciardullo A, et al. Varicose veins of the lower limbs and venous capacitance in postmenopausal women: relationship with obesity. J Vasc Surg. 2002;36:965-8.

12. Beebe-Dimmer JL, Pfeifer JR, Engle JS, Schottenfeld D. The epidemiology of chronic venous insufficiency and varicose veins. Ann Epidemiol. 2005;15:175-84.

13. Fowkes FGR, Lee AJ, Evans C), Allan PL, Bradbury AW, Ruckley CV. Lifestyle risk factors for lower limb venous reflux in the general population: Edinburgh Vein Study. Int J Epidemiol. 2001;30:846-52.

14. Lionis C, Erevnidou K, Argyriadou S, Vlachonikolis I, Katsamouris A, Antonakis N; CVI Research Group. Chronic venous insufficiency. A common health problem in general practice in Grecce. Int Angiol. 2002;21:86-92.

15. Ruckley CV, Evans C), Allan PL, Lee AJ, Fowkes FGR. Chronic venous insufficiency: clinical and duplex correlations. The Edinburgh Vein Study of venous disorders in the general population. J Vasc Surg. 2002;36:520-5.
16. Raju N, Neglén P. Chronic Venous Insufficiency and Varicose Veins. N Engl J Med. 2009;360:2319-27.

17. Safar H, Shawa N, Al-Ali J, Al-Nassar M, Dashti H, Asfar S. Is there a need for Doppler vascular examination for the diagnosis of varicose vein? A prospective study. Med Princ Pract. 2004;13:43-6.

18. van Bemmelen PS, Bedford G, Beach K, Strandness DE. Quantitative segmental evaluation of venous valvular reflux with duplex ultrasound scanning. I Vasc Surg. 1989;10:425-31.

19. Fowkes FGR, Evans C), Lee AJ. Prevalence and risk factors of chronic venous insufficiency. Angiology. 2001;52:S5-S15.

20. Kurz X, Kahn SR, Abenhaim L, et al. Chronic venous disorders of the leg: epidemiology, outcomes, diagnosis and management. Summary of an evidence-based report of the VEINES task force. Venous Insufficiency Epidemiologic and Economic Studies. Int Angiol. 1999;18:83-102.

21. Sugerman HJ, Sugerman EL, Wolfe L, et al. Risks and benefits of gastric bypass in morbidly obese patients with severe venous stasis disease. Ann Surg. 2001;234:41-6.

22. Padberg F Jr, Cerveira JJ, Lal BK, Pappas PJ, Varma S, Hobson RW 2nd. Does severe venous insufficiency have a different etiology in the morbidly obese? Is it venous? J Vasc Surg. 2003;37:79-85.

23. Porter JM, Moneta GL. Reporting standards in venous disease: an update. International Consensus Committee on Chronic Venous Disease. J Vasc Surg. 1995;21:635-45.

24. van Rij AM, De Alwis CS, Jiang P, et al. Obesity and impaired venous function. Eur J Vasc Endovasc Surg. 2008;35:739-44.

25. Danielsson G, Eklof B, Grandinetti A, Kistner RL. The influence of obesity on chronic venous disease. Vasc Endovascular Surg. 2002;36:271-6.

26. Scott TE, LaMorte WW, Gorin DR, Menzoian JO. Risk factor for chronic venous insufficiency: a dual case-control study. J Vasc Surg. 1995;22:622-8.

27. Brand FN, Dannenberg AL, Abbott RD, Kannell WB. The epidemiology of varicose veins: the Framingham study. Am J Prev Med. 1988;4:96-101.

28. Evans (]), Fowkes FGR, Ruckley CV, Lee AJ. Prevalence of varicose vein and chronic venous insufficiency in men and women in the general population: Edinburg Vein Study. J Epidemiol Community Health. 1999;53:149-53.

29. Mekky S, Schilling RSF, Walford J. Varicose veins in women cotton workers. An epidemiological study in England and Egypt. Br Med J. 1969;2:591-5.

30. Sisto T, Reunanen A, Laurikka J, et al. Prevalence and risk factors of varicose veins in lower extremities: mini-Finland health survey. Eur J Surg. 1995; 161:405-14.

31. Seidell JC, de Groot L, van Sonsbeek JL, Deurenberg P, Hautvast J. Associations of moderate and severe overweight with self-reported illness and medical care in Dutch adults. Am J Public Health. 1986;76:264-9.

32. Seidell JC, Bakx KC, Deurenberg P, van den Hoogen H, Hautvast J, Stijnen T. Overweight and chronic illness--a retrospective cohort study, with a follow-up of 6-17 years, in men and women of initially 20-50 years of age. J Chronic Dis. 1986;39:585-93.

33. van Noord PA, Seidell JC, den Tonkelaar I, Baanders-van Halewijn EA, Ouwehand II. The relationship between fat distribuition and 
some chronic disease in 11,825 women participating in the DOMproject. Int J Epidemiol. 1990;19:564-70.

34. Stanhope JM. Varicose veins in a population of lowland New Guinea. Int J Epidemiol. 1975;4:221-5.

35. Malhotra SL. An epidemiological study of varicose veins in Indian railroad workers from the South and North of India, with special reference to the causation and prevention of varicose veins. Int J Epidemiol. 1972;1:177-83.

36. Robertson L, Evans C, Fowkes FG. Epidemiology of chronic venous disease. Phlebology. 2008;23:103-11.

37. Kostas TI, loannou CV, Drygiannakis I, et al. Chronic venous disease progression and modification of predisposing factors. J Vasc Surg. 2010;51:900-7.

38. Lemaire R. The flow of venous blood in the obese. Phebologie. 1988;41:493-9.

39. Pitta GBB. Cirurgia de varizes na obesidade. In: Pitta GBB, Castro $A A$, Burihan E, editors. Angiologia e cirurgia vascular: guia ilustrado. Maceió: UNCISAL/ECMAL \& LAVA; 2003.

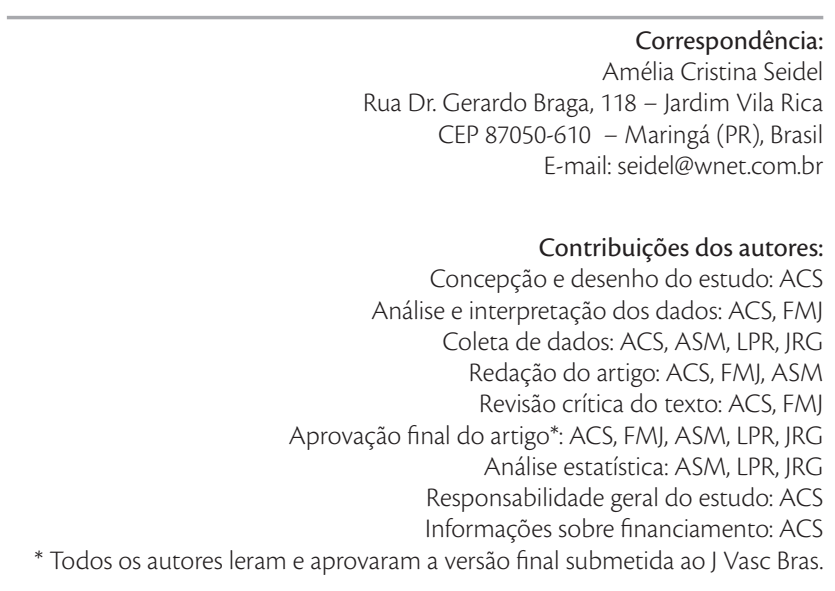

